\title{
THE CURVATURE OF SEMIDIRECT PRODUCT GROUPS ASSOCIATED WITH TWO-COMPONENT HUNTER-SAXTON SYSTEMS
}

\author{
MARTIN KOHLMANN
}

\begin{abstract}
In this paper, we study two-component versions of the periodic Hunter-Saxton equation and its $\mu$-variant. Considering both equations as a geodesic flow on the semidirect product of the circle diffeomorphism group $\operatorname{Diff}(\mathbb{S})$ with a space of scalar functions on $\mathbb{S}$ we show that both equations are locally well-posed. The main result of the paper is that the sectional curvature associated with the $2 \mathrm{HS}$ is constant and positive and that $2 \mu \mathrm{HS}$ allows for a large subspace of positive sectional curvature. The issues of this paper are related to some of the results for $2 \mathrm{CH}$ and $2 \mathrm{DP}$ presented in [1].
\end{abstract}

\section{Introduction}

The Hunter-Saxton (HS) equation

$$
u_{t x x}+2 u_{x} u_{x x}+u u_{x x x}=0, \quad x \in \mathbb{S}, \quad t>0,
$$

was derived in [18] as a model for propagation of orientation waves in a massive nematic liquid crystal director field. The function $u(t, x)$ depends on a space variable $x$ and a slow time variable $t$. In recent years, the question of the existence and regularity of solutions to (1) as well as integrability properties have been examined in great detail, cf., e.g., [3, 6 , 19, 37]. The HS equation can be regarded as the $\alpha \rightarrow \infty$-limit of the Camassa-Holm (CH) equation

$$
m_{t}=-\left(m_{x} u+2 m u_{x}\right), \quad m=\left(1-\alpha \partial_{x}^{2}\right) u=u-\alpha u_{x x}
$$

which was introduced to model the shallow-water medium-amplitude regime for wave motion over a flat bad, [4]; more precisely, the HS equation is equivalent to

$$
m_{t}=-\left(m_{x} u+2 m u_{x}\right), \quad m=\left(-\partial_{x}^{2}\right) u=-u_{x x} .
$$

Alternatively, the HS equation can be regarded as the high-frequency or short-wave limit $(x, t) \mapsto(\varepsilon x, \varepsilon t)$, for $\varepsilon \rightarrow 0$, of the $\mathrm{CH}$ equation, cf. [9, 19]. Similarly to the $\mathrm{CH}$, the HS equation comes up from Langrange's variational principle with the Lagrangian

$$
\mathcal{L}_{H S}(u)=\frac{1}{2} \int_{\mathbb{S}} u_{x}^{2} \mathrm{~d} x
$$

cf. [19], which differs from the Lagrangian for $\mathrm{CH}$ only in a term proportional to $u^{2}$ under the integral sign. The Euler-Lagrange equation for the modified Lagrangian

$$
\mathcal{L}_{2 H S}(u, \rho)=\frac{1}{2} \int_{\mathbb{S}} u_{x}^{2} \mathrm{~d} x+\frac{1}{2} \int_{\mathbb{S}} \rho^{2} \mathrm{~d} x
$$

Date: March 8, 2018.

2000 Mathematics Subject Classification. 37K65, 58B25, 58D05.

Key words and phrases. Hunter-Saxton equation, semidirect product, geodesic flow, curvature. 
is the $2 \mathrm{HS}$ equation

$$
\left\{\begin{array}{l}
m_{t}=-m_{x} u-2 m u_{x}-\rho \rho_{x} \\
\rho_{t}=-(\rho u)_{x}
\end{array}\right.
$$

an integrable two-component extension of (1) which reduces to (1) for $\rho=0$.

We note that the two-component HS equation is a particular case of the GurevichZybin system pertaining to nonlinear one-dimensional dynamics of dark matter as well as nonlinear ion-acoustic waves, cf. [33]. Also, it is related to the twocomponent Camassa-Holm $(2 \mathrm{CH})$ system (which reads (3) with $m=u-u_{x x}$, 7, 13]) via the short-wave limit. Note that for the $2 \mathrm{CH}$ equation, the second variable $\rho$ can be interpreted as the fluid's density and its evolution equation in fact is the continuity equation for velocity and fluid density. The two-component Hunter-Saxton (3) equation is a special case of the two-parameter family of evolution equations

$$
\left\{\begin{aligned}
m_{t} & =-m_{x} u-a u_{x} m-\kappa \rho \rho_{x}, \\
m & =-u_{x x}, \quad(a, \kappa) \in \mathbb{R}^{2}, \\
\rho & =-\rho u_{x}+(1-a) u_{x} \rho,
\end{aligned}\right.
$$

from which it emerges for the choice $(a, \kappa)=(2,1)$. The system (4) is of great importance for a variety of problems occurring in mathematical physics: A special case of the two-parameter family (4) is the Proudman-Johnson equation [32, 34] for $\rho=0$ and $a=-1$; this equation is obtained from the incompressible 2D Euler equations by a special ansatz for the stream function. Further relations to, e.g., the inviscid Kármán-Batchelor flow [5, 17], which derives from the 3D axisymmetric Euler equations, or the famous Constantin-Lax-Majda equation [8] are explained in [35]. We also refer the reader to [35] for an extensive discussion of well-posedness and blow-up for the general system (4).

What makes the 2HS system particularly interesting is its potential exhibition of nonlinear phenomena as breaking waves and peakons [7]. In 21] the authors derive the $2 \mathrm{HS}$ equation as the $N=2$ supersymmetric extension of the CamassaHolm equation. They also work out the bi-Hamiltonian formulation and a Lax pair representation for the $2 \mathrm{HS}$ equation. Concerning geometry, the 2HS equation can be regarded as an Euler equation on the superconformal algebra of contact vector fields on the 1|2-dimensional supercircle. Finally, 21] show some explicit solutions of (3), like bounded traveling waves.

Interesting variants of Eqs. (11) and (3) are obtained by using $m=\mu(u)-u_{x x}$ instead of $m=-u_{x x}$ in (2) and (3), where $\mu(u)$ is the mean of $u: \mathbb{S} \rightarrow \mathbb{R}$. This way we obtain the $\mu \mathrm{HS}$ equation

$$
0=u_{t x x}+2 u_{x} u_{x x}+u u_{x x x}-2 \mu(u) u_{x}
$$

and its two-component extension

$$
\left\{\begin{array}{l}
0=u_{t x x}+2 u_{x} u_{x x}+u u_{x x x}-2 \mu(u) u_{x}-\rho \rho_{x} \\
\rho_{t}=-(\rho u)_{x}
\end{array}\right.
$$

In 20] it is explained that the $\mu \mathrm{HS}$ equation models nematic liquid crystals with a preferred direction of the director field, e.g., coming from an external magnetic field acting on dipoles and turning them to this direction. Our motivation for studying the 2-component extension $2 \mu \mathrm{HS}$ comes from the observation that the generalized system possesses an integrable structure [38] and the expectancy that 
similar relationships between $\mu \mathrm{HS}$ and $2 \mu \mathrm{HS}$ compared to HS and 2HS might hold true. Both, (5) and (6), are integrable equations related to the isospectral problem

$$
\left\{\begin{array}{ccc}
\psi_{x x}+\left(m \lambda+\rho^{2} \lambda^{2}\right) \psi & = & 0 \\
\psi_{t} & = & -\left(\frac{1}{2 \lambda}+u\right) \psi_{x}+\frac{1}{2} \psi u_{x}
\end{array}\right.
$$

i.e., $\psi_{t x x}=\psi_{x x t}$ and $\lambda_{t}=0$ imply the $2 \mu \mathrm{HS}$ equation (6). Equations (5) and (6) follow from the variational principle with the Lagrangians

$$
\mathcal{L}_{\mu H S}(u)=\frac{1}{2} \int_{\mathbb{S}} u\left(\mu-\partial_{x}^{2}\right) u \mathrm{~d} x
$$

and

$$
\mathcal{L}_{2 \mu H S}(u, \rho)=\frac{1}{2} \int_{\mathbb{S}} u\left(\mu-\partial_{x}^{2}\right) u \mathrm{~d} x+\frac{1}{2} \int_{\mathbb{S}} \rho^{2} \mathrm{~d} x .
$$

While there are some well-posedness results for the $\mu \mathrm{HS}$ in [20], there is no fully developed existence and regularity theory for its two-component version up to now. A first attempt to construct global weak solutions is presented in [28].

We now turn to the geometric theory behind (1), (3), (5) and (6) which will be relevant for this paper: Equations (1) and (5) have been studied as geodesic flow on the circle diffeomorphism group $H^{s} \operatorname{Diff}(\mathbb{S})$ of Sobolev class $H^{s}$, 23, 20]. We provide some elementary results about recasting Euler equations as geodesic equations on infinite-dimensional Lie groups in an appendix of this paper; see Section 5 . The strategy is to define a right-invariant metric on $H^{s} \operatorname{Diff}(\mathbb{S})$, induced at the identity by the inertia operators $-\partial_{x}^{2}$ and $\mu-\partial_{x}^{2}$ respectively, and to prove its compatibility with an affine connection which is given canonically in terms of the Christoffel operator for the respective equation. This implies the existence of a geodesic flow and first local well-posedness result 1 . Moreover, computations of the curvature tensor and the sectional curvature for both equations have been performed. The meaningful results are that the sectional curvature is strictly positive for both equations and that HS has constant positive sectional curvature which motivates that, in this case, $H^{s} \operatorname{Diff}(\mathbb{S})$ is locally isometric to an $L_{2}$-sphere. This sphere interpretation has various geometric consequences as explained in [24, 25]. Furthermore, the positivity of sectional curvatures is related to stability issues for the geodesics, cf. Section 5 .

In this paper, we extend some of the above results to the integrable 2-component extensions (3) and (6). In a first step we comment on how to choose a suitable configuration manifold for $2 \mathrm{HS}$ and $2 \mu \mathrm{HS}$; here, we will make use of semidirect products. Then we give a proof of the fact that the curvature tensor for $2 \mathrm{HS}$ is of the same form as for HS with the only difference that the geometric objects contained therein have to be replaced by their two-component analogs. For $2 \mu \mathrm{HS}$ we first obtain a curvature formula which is similar to the formula for the one-component $\mu \mathrm{HS}$. Second, we compute the sectional curvature in several two-dimensional directions and establish a positivity result. The paper is organized as follows:

Section 2 explains the geometric structure of $2 \mathrm{HS}$ and $2 \mu \mathrm{HS}$; here we first recall some results about the corresponding one-component equations and semidirect products which we will need in the following. The main goal of this section is to establish the existence of a unique local geodesic flow and local well-posedness for both equations. Section 3 is about the curvature of the product group associated with the 2HS. In Section 4 we express the sectional curvature for $2 \mu \mathrm{HS}$ in terms of its Christoffel map and present a large subspace of positive sectional curvature.

\footnotetext{
${ }^{1}$ For HS one has to factorize $H^{s} \operatorname{Diff}(\mathbb{S})$ by a subgroup as we will explain in the following.
} 


\section{Geometric ASPeCts of $2 \mathrm{HS}$ And $2 \mu \mathrm{HS}$}

Let us denote by $H^{s} \operatorname{Diff}(\mathbb{S})$ the group of orientation-preserving $H^{s}$ diffeomorphisms of $\mathbb{S}=\mathbb{R} / \mathbb{Z}$ where $H^{s}=H^{s}(\mathbb{S})$ is the Sobolev space of order $s>0$ on the circle. Note that, for any $s>3 / 2, H^{s} \operatorname{Diff}(\mathbb{S})$ is a topological group and a smooth Hilbert manifold modelled on the space $H^{s}$, but not a Lie group, [23, 31]. We have $T H^{s} \operatorname{Diff}(\mathbb{S}) \simeq H^{s} \operatorname{Diff}(\mathbb{S}) \times H^{s}(\mathbb{S})$.

2.1. The HS equation. Let us rewrite the HS equation as an autonomous system in terms of the local flow $X(t)=\left(\varphi(t), \varphi_{t}(t)\right) \in T H^{s} \operatorname{Diff}(\mathbb{S}), s>5 / 2$, for the timedependent $H^{s}$ vector field $u(t, \cdot)$ on $\mathbb{S}$. The usual starting point is to compute $\varphi_{t t}$. By the chain rule and from the relation $\varphi_{t}=u \circ \varphi$, we obtain

$$
\varphi_{t t}=\left(u_{t}+u u_{x}\right) \circ \varphi .
$$

We now have to replace $u_{t}$ by using Eq. (11). But since Eq. (1) only includes $u_{t x x}$, we differentiate twice with respect to $x$ to obtain

$$
\begin{aligned}
\partial_{x}^{2}\left(u_{t}+u u_{x}\right) & =u_{t x x}+3 u_{x} u_{x x}+u u_{x x x} \\
& =u_{x} u_{x x} \\
& =\frac{1}{2} \partial_{x} u_{x}^{2} .
\end{aligned}
$$

Now we are in need of the inverse of the operator $A=-\partial_{x}^{2}$.

Lemma 1. Let $A$ be the operator $-\partial_{x}^{2}$ with domain

$$
D(A)=\left\{f \in H^{s}(\mathbb{S}) ; f(0)=0\right\}, \quad s>5 / 2 .
$$

For any $s>5 / 2, A$ is a topological isomorphism

$$
D(A) \rightarrow\left\{f \in H^{s-2}(\mathbb{S}) ; \int_{\mathbb{S}} f(x) \mathrm{d} x=0\right\}
$$

with the inverse

$$
\left(A^{-1} f\right)(x)=-\int_{0}^{x} \int_{0}^{y} f(z) \mathrm{d} z \mathrm{~d} y+x \int_{\mathbb{S}} \int_{0}^{y} f(z) \mathrm{d} z \mathrm{~d} y .
$$

By Sobolev's imbedding theorem $u_{x} \in C(\mathbb{S})$ and hence the right-hand side of (7) is a function with zero mean. Thus it is in the domain of $A^{-1}$ and we conclude

$$
\varphi_{t t}=-\frac{1}{2}\left[A^{-1} \partial_{x}\left(\varphi_{t} \circ \varphi^{-1}\right)_{x}^{2}\right] \circ \varphi .
$$

Note also that $u_{t}+u u_{x}$ must belong to the domain of $A$ which suggests that we will need the assumption $u(0)=0$. Setting

$$
\Gamma(u, v)=-\frac{1}{2} A^{-1}\left(u_{x} v_{x}\right)_{x},
$$

we obtain a symmetric bilinear operator $H^{s}(\mathbb{S}) \times H^{s}(\mathbb{S}) \rightarrow H^{s}(\mathbb{S})$. In fact, this Christoffel map is smooth which enables the following geometric approach, established in [25]:

Let $\operatorname{Rot}(\mathbb{S}) \subset H^{s} \operatorname{Diff}(\mathbb{S})$ be the subgroup of rotations $x \mapsto x+d$ for some $d \in \mathbb{R}$. We denote by $H^{s} \operatorname{Diff}(\mathbb{S}) / \operatorname{Rot}(\mathbb{S})$ the space of right cosets $\operatorname{Rot}(\mathbb{S}) \circ \varphi=$ $\{\varphi(\cdot)+d ; d \in \mathbb{R}\}$ for $\varphi \in H^{s} \operatorname{Diff}(\mathbb{S})$ and set $M^{s}=\left\{\varphi \in H^{s} \operatorname{Diff}(\mathbb{S}) ; \varphi(0)=0\right\}$. We have

$$
M^{s}=\left\{\mathrm{id}+u ; u \in H^{s}, u_{x}>-1, u(0)=0\right\}
$$


and thus $M^{s}$ is an open subset of the closed hyperplane

$$
\mathrm{id}+E^{s}:=\mathrm{id}+\left\{u \in H^{s} ; u(0)=0\right\} \subset H^{s} .
$$

Writing the elements of $H^{s} \operatorname{Diff}(\mathbb{S}) / \operatorname{Rot}(\mathbb{S})$ as $[\varphi]$, the map $[\varphi] \mapsto \varphi-\varphi(0)$ establishes a diffeomorphism $H^{s} \operatorname{Diff}(\mathbb{S}) / \operatorname{Rot}(\mathbb{S}) \rightarrow M^{s}$, showing in this way that $M^{s}$ is a global chart for $H^{s} \operatorname{Diff}(\mathbb{S}) / \operatorname{Rot}(\mathbb{S})$. Furthermore, all tangent spaces $T_{\varphi} M^{s}$ can be identified with $E^{s}$. Next, we define a right-invariant metric on $H^{s} \operatorname{Diff}(\mathbb{S}) / \operatorname{Rot}(\mathbb{S})$ by setting

$$
\langle U, V\rangle_{\varphi}=\frac{1}{4} \int_{\mathbb{S}}\left(U \circ \varphi^{-1}\right) A\left(V \circ \varphi^{-1}\right) \mathrm{d} x=\frac{1}{4} \int_{\mathbb{S}} \frac{U_{x} V_{x}}{\varphi_{x}} \mathrm{~d} x
$$

for tangent vectors $U, V \in T_{\varphi} M^{s} \simeq E^{s}$ at $\varphi \in M^{s}$. Recall that the bilinear form $\langle\cdot, \cdot\rangle_{\text {id }}$ at the identity, induced by the operator $A$ defined in Lemma 1, is the $\dot{H}^{1}$ metric and that our definition of $A$ ensures that $\langle\cdot, \cdot\rangle_{\text {id }}$ is indeed a positive definite inner product2. Furthermore the metric (9) is compatible with the affine connection $\nabla$ defined locally by

$$
\nabla_{X} Y(\varphi)=D Y(\varphi) \cdot X(\varphi)-\Gamma(\varphi ; Y(\varphi), X(\varphi))
$$

where $\Gamma(\varphi ; \cdot, \cdot)=R_{\varphi} \circ \Gamma(\mathrm{id}, \cdot, \cdot) \circ R_{\varphi}^{-1}$ is the smooth Christoffel map for the HS equation with $\Gamma(\mathrm{id}, u, v)=-\frac{1}{2} A^{-1}\left(u_{x} v_{x}\right)_{x}$. As proved in [25], the geodesics of the $\dot{H}^{1}$ right-invariant metric are described by the HS equation: Let $J \subset \mathbb{R}$ be an open interval and let $\varphi: J \rightarrow H^{s} \operatorname{Diff}(\mathbb{S})$ be a smooth curve. Then the curve $u: J \rightarrow T_{\text {id }} H^{s}$ Diff( $(\mathbb{S})$ defined by $u: t \mapsto \varphi_{t} \circ \varphi^{-1}$ satisfies the HS equation (1) if and only if the curve $[\varphi]: J \rightarrow H^{s} \operatorname{Diff}(\mathbb{S}) / \operatorname{Rot}(\mathbb{S})$ given by $[\varphi]: t \mapsto[\varphi(t)]$ is a geodesic with respect to $\nabla$. The geodesics in $H^{s} \operatorname{Diff}(\mathbb{S}) / \operatorname{Rot}(\mathbb{S})$ can be found explicitly by the method of characteristics: For $u_{0} \in T_{\mathrm{id}} M^{s}$ with $\left\langle u_{0}, u_{0}\right\rangle=1$ the unique geodesic $\varphi:\left[0, T^{*}\left(u_{0}\right)\right) \rightarrow M^{s}$ with $\varphi(0)=$ id and $\varphi_{t}(0)=u_{0}$ is given by

$$
\varphi(t)=\mathrm{id}-\frac{1}{8}\left(A^{-1} \partial_{x}\left(u_{0 x}^{2}\right)\right)(1-\cos 2 t)+\frac{1}{2} u_{0} \sin 2 t,
$$

where the maximal time of existence is

$$
T^{*}\left(u_{0}\right)=\frac{\pi}{2}+\arctan \left(\frac{1}{2} \min _{x \in \mathbb{S}} u_{0 x}(x)\right)<\pi / 2 .
$$

Observe that the associated solution $u=\varphi_{t} \circ \varphi^{-1} \in C\left(\left[0, T^{*}\right) ; E^{s}\right) \cap C^{1}\left(\left[0, T^{*}\right) ; E^{s-1}\right)$ of the HS is not unique; the set of solutions is

$$
\left\{t \mapsto u(t, \cdot-c(t))+c^{\prime}(t)\right\} \subset C\left([0, T) ; H^{s}(\mathbb{S})\right) \cap C^{1}\left([0, T) ; H^{s-1}(\mathbb{S})\right),
$$

where $T \leq T^{*}$ is the maximal time of existence, $c:[0, T) \rightarrow \mathbb{R}$ is an arbitrary $C^{1}$ function with $c(0)=c^{\prime}(0)=0$ and if $T<T^{*}$, then $|c(t)| \rightarrow \infty$ as $t \rightarrow T$ from below. Further geometric aspects of the HS equation are discussed in [23, 24].

2.2. The $\mu$ HS equation. The inertia operator for $\mu \mathrm{HS}$ is $A=\mu-\partial_{x}^{2}$, where $\mu(u)=\int_{\mathbb{S}} u \mathrm{~d} x$. The following lemma can be found in [27].

\footnotetext{
${ }^{2}$ The factor $1 / 4$ is introduced to obtain that the sectional curvature for HS is identically equal to one.
} 
Lemma 2. For $s \geq 2$, the linear operator $A=\mu-\partial_{x}^{2}: H^{s} \rightarrow H^{s-2}$ is a topological isomorphism with the inverse

$$
\begin{aligned}
\left(A^{-1} f\right)(x)= & \left(\frac{1}{2} x^{2}-\frac{1}{2} x+\frac{13}{12}\right) \int_{0}^{1} f(a) \mathrm{d} a+\left(x-\frac{1}{2}\right) \int_{0}^{1} \int_{0}^{a} f(b) \mathrm{d} b \mathrm{~d} a \\
& -\int_{0}^{x} \int_{0}^{a} f(b) \mathrm{d} b \mathrm{~d} a+\int_{0}^{1} \int_{0}^{a} \int_{0}^{b} f(c) \mathrm{d} c \mathrm{~d} b \mathrm{~d} a
\end{aligned}
$$

and the Green's function

$$
\left(A^{-1} f\right)(x)=\int_{\mathbb{S}} g\left(x-x^{\prime}\right) f\left(x^{\prime}\right) \mathrm{d} x^{\prime}, \quad g\left(x-x^{\prime}\right)=\frac{1}{2}\left(x-x^{\prime}\right)^{2}-\frac{1}{2}\left|x-x^{\prime}\right|+\frac{13}{12} .
$$

Let $s>5 / 2$. The Christoffel operator $\Gamma=\Gamma_{\text {id }}$ for Eq. (5) is

$$
\Gamma(u, v)=-A^{-1}\left(\mu(u) v+\mu(v) u+\frac{1}{2} u_{x} v_{x}\right)_{x},
$$

since $\mu \mathrm{HS}$ can be written as $u_{t}+u u_{x}=\Gamma(u, u)$. The bilinear map

$$
\langle U, V\rangle_{\varphi}=\mu\left(U \circ \varphi^{-1}\right) \mu\left(V \circ \varphi^{-1}\right)+\int_{\mathbb{S}} \frac{U_{x} V_{x}}{\varphi_{x}} \mathrm{~d} x, \quad U, V \in T_{\varphi} H^{s} \operatorname{Diff}(\mathbb{S})
$$

defines a right-invariant inner product on $H^{s} \operatorname{Diff}(\mathbb{S})$ and the pair $\left(H^{s} \operatorname{Diff}(\mathbb{S}),\langle\cdot, \cdot\rangle\right)$ is a Riemannian manifold. The $\mu$ HS Christoffel map depends smoothly on $\varphi$ and defines a Riemannian covariant derivative on $H^{s} \operatorname{Diff}(\mathbb{S})$ via (10), compatible with the right-invariant metric $\langle\cdot, \cdot\rangle$. In consequence, the $\mu \mathrm{HS}$ possesses a unique geodesic flow $\varphi \in H^{s} \operatorname{Diff}(\mathbb{S})$. As a geodesic equation on $H^{s} \operatorname{Diff}(\mathbb{S})$, the $\mu \mathrm{HS}$ reads $\varphi_{t t}=$ $\Gamma_{\varphi}\left(\varphi_{t}, \varphi_{t}\right)$ in Lagrangian coordinates. We also conclude that $\mu \mathrm{HS}$ is locally wellposed in $H^{s}$ for $s>5 / 2$, cf. [20].

2.3. Generalities on semidirect products. Let $G$ be a Lie group and $V$ be a vector space. If $G$ acts on the right on $V$, one defines

$$
\left(g_{1}, v_{1}\right)\left(g_{2}, v_{2}\right)=\left(g_{1} g_{2}, v_{2}+v_{1} g_{2}\right)
$$

and with this product, $G \times V$ becomes a Lie group (the semidirect product of $G$ and $V)$ which is denoted as $G(S)$. It is easy to see that $(e, 0)$ is the neutral element, where $e$ denotes the neutral element of $G$, and that $(g, v)$ has the inverse $\left(g^{-1},-v g^{-1}\right)$. To obtain the Lie bracket on the Lie algebra $\mathfrak{g} \mathrm{S} V$, we consider the inner automorphism

$$
I_{(g, v)}(h, w)=(g, v)(h, w)(g, v)^{-1}=\left(g h g^{-1},-v g^{-1}+(w+v h) g^{-1}\right) .
$$

Writing $v \xi$ for the induced infinitesimal action of $\mathfrak{g}$ on $V$, i.e., the map

$$
V \times \mathfrak{g} \mapsto V, \quad(v, \xi) \mapsto v \xi:=\left.\frac{\mathrm{d}}{\mathrm{d} t} v g(t)\right|_{t=0},
$$

$g(t)$ being a curve in $G$ starting from $e$ in the direction of $\xi$, we obtain

$$
\begin{gathered}
\operatorname{Ad}_{(g, v)}(\xi, w)=\left(\operatorname{Ad}_{g} \xi,(w+v \xi) g^{-1}\right), \\
\operatorname{ad}_{(\eta, v)}(\xi, w)=\left(\operatorname{ad}_{\eta} \xi, v \xi-w \eta\right)
\end{gathered}
$$

and hence

$$
\left[\left(\xi_{1}, v_{1}\right),\left(\xi_{2}, v_{2}\right)\right]=\operatorname{ad}_{\left(\xi_{2}, v_{2}\right)}\left(\xi_{1}, v_{1}\right)=\left(\left[\xi_{1}, \xi_{2}\right], v_{2} \xi_{1}-v_{1} \xi_{2}\right)
$$

For our purposes, we consider the semidirect product of the orientation-preserving diffeomorphisms $H^{s} \operatorname{Diff}(\mathbb{S})$ with $H^{s-1}$; the structure of our equations motivates to enforce the second component to have one order less regularity than the first, cf. also 
11]. We will use the notation $H^{s} G$ and $H^{s} G_{0}$ for the Lie groups $H^{s} \operatorname{Diff}(\mathbb{S})\left(S H^{s-1}(\mathbb{S})\right.$ and $\left[H^{s} \operatorname{Diff}(\mathbb{S}) / \operatorname{Rot}(\mathbb{S})\right]\left(S E^{s-1}(\mathbb{S})\right.$. The group product in these groups is defined by

$$
\left(\varphi_{1}, f_{1}\right)\left(\varphi_{2}, f_{2}\right):=\left(\varphi_{1} \circ \varphi_{2}, f_{2}+f_{1} \varphi_{2}\right)
$$

where $\circ$ denotes the group product in $H^{s} \operatorname{Diff}(\mathbb{S})$ (i.e., composition) and $f \varphi:=f \circ \varphi$ is a right action of $H^{s} \operatorname{Diff}(\mathbb{S})$ on the scalar functions on $\mathbb{S}$. The neutral element is (id, 0$)$ and $(\varphi, f)$ has the inverse $\left(\varphi^{-1},-f \circ \varphi^{-1}\right)$. The above calculations show that

$$
\begin{gathered}
\operatorname{Ad}_{(\varphi, f)}(u, \rho)=\left(\operatorname{Ad}_{\varphi} u,\left(f_{x} u+\rho\right) \circ \varphi^{-1}\right), \\
\operatorname{ad}_{(v, f)}(u, \rho)=\left(\operatorname{ad}_{v} u, f_{x} u-\rho_{x} v\right)
\end{gathered}
$$

and

$$
\left[\left(u_{1}, u_{2}\right),\left(v_{1}, v_{2}\right)\right]=\left(\left[u_{1}, v_{1}\right], v_{2 x} u_{1}-u_{2 x} v_{1}\right),
$$

where $\left[u_{1}, v_{1}\right]=v_{1 x} u_{1}-u_{1 x} v_{1}$ is the Lie bracket induced by right-invariant vector fields on $H^{s} \operatorname{Diff}(\mathbb{S})$. Observe that $T H^{s} G \simeq H^{s} G \times\left(H^{s} \times H^{s-1}\right)$ and $T H^{s} G_{0} \simeq$ $H^{s} G_{0} \times\left(E^{s} \times E^{s-1}\right)$. For further details about semidirect product groups we refer to 15,16$]$.

2.4. The $2 \mathrm{HS}$ equation. Inspired by the results for $2 \mathrm{CH}$ and $2 \mathrm{DP}$ in [11], we work with the configuration space

$$
H^{s} G_{0}=\left[H^{s} \operatorname{Diff}(\mathbb{S}) / \operatorname{Rot}(\mathbb{S})\right]\left(S E^{s-1}(\mathbb{S}), \quad s>5 / 2 .\right.
$$

The Lagrangian for 2HS motivates to define the right-invariant metric on $H^{s} G_{0}$ which equals the $\dot{H}^{1}$-metric for the first component plus the $L_{2}$-metric for the second component at the identity (id, 0 ):

$$
\langle U, V\rangle_{(\varphi, f)}=\left\langle u_{1} \circ \varphi^{-1}, v_{1} \circ \varphi^{-1}\right\rangle_{\dot{H}^{1}}+\left\langle u_{2} \circ \varphi^{-1}, v_{2} \circ \varphi^{-1}\right\rangle_{L_{2}},
$$

for $U=\left(u_{1}, u_{2}\right), V=\left(v_{1}, v_{2}\right) \in T_{(\varphi, f)} H^{s} G_{0}$. Let $A$ and $\Gamma^{0}$ as in Lemma 1 and (8)). With the 2HS Christoffel map $\Gamma$ on $E^{s} \times E^{s-1}$ given by

$$
\Gamma(X, Y)=\left(\begin{array}{c}
\Gamma_{\mathrm{id}}^{0}\left(X_{1}, Y_{1}\right)-\frac{1}{2} A^{-1}\left(X_{2} Y_{2}\right)_{x} \\
-\frac{1}{2}\left(X_{1 x} Y_{2}+Y_{1 x} X_{2}\right)
\end{array}\right),
$$

we define the map $\Gamma:\left(M^{s} \times E^{s-1}\right) \times\left(E^{s} \times E^{s-1}\right)^{2} \rightarrow E^{s} \times E^{s-1}$,

$$
\Gamma_{(\varphi, f)}(X, Y)=\Gamma((\varphi, f) ; X, Y)=\Gamma\left(X \circ \varphi^{-1}, Y \circ \varphi^{-1}\right) \circ \varphi .
$$

Finally, we introduce the affine connection

$$
\nabla_{X} Y(\varphi, f)=D Y(\varphi, f) \cdot X(\varphi, f)-\Gamma((\varphi, f) ; Y(\varphi, f), X(\varphi, f)) .
$$

The proof of the following proposition uses standard arguments and it is omitted for the reader's convenience.

Proposition 3. Let $s>5 / 2$. Let $H^{s} G_{0}=\left[H^{s} \operatorname{Diff}(\mathbb{S}) / \operatorname{Rot}(\mathbb{S})\right](S) E^{s-1}(\mathbb{S})$ and let $\Gamma$ be the Christoffel map defined in (13) and (14). Then $\Gamma$ defines a smooth spray on $H^{s} G_{0}$, i.e., the map

$$
([\varphi], f) \mapsto \Gamma_{([\varphi], f)}: H^{s} G_{0} \rightarrow \mathcal{L}_{\text {sym }}^{2}\left(E^{s} \times E^{s-1} ; E^{s} \times E^{s-1}\right)
$$

is smooth. Moreover, the metric $\langle\cdot, \cdot\rangle$ defined in (12) is a smooth (weak) Riemannian metric on $H^{s} G_{0}$, i.e., the map

$$
([\varphi], f) \mapsto\langle\cdot, \cdot\rangle_{([\varphi], f)}: H^{s} G_{0} \rightarrow \mathcal{L}_{\text {sym }}^{2}\left(T_{([\varphi], f)} H^{s} G_{0} ; \mathbb{R}\right)
$$


is a smooth section of the bundle $\mathcal{L}_{\mathrm{sym}}^{2}\left(T H^{s} G_{0} ; \mathbb{R}\right)$. Finally, the connection $\nabla$ and the metric $\langle\cdot, \cdot\rangle$ are compatible in the sense that

$$
X\langle Y, Z\rangle=\left\langle\nabla_{X} Y, Z\right\rangle+\left\langle Y, \nabla_{X} Z\right\rangle
$$

for all vector fields $X, Y, Z$ on $H^{s} G_{0}$.

As a consequence, we obtain a unique local geodesic flow $(\varphi(t), f(t)) \in H^{s} G_{0}$ for 2 HS satisfying

$$
\left(\varphi_{t t}, f_{t t}\right)=\Gamma_{(\varphi, f)}\left(\left(\varphi_{t}, f_{t}\right),\left(\varphi_{t}, f_{t}\right)\right) .
$$

Theorem 4. Let $s>5 / 2$. Then there exists an open interval $J$ centered at 0 and an open neighborhood $U$ of $(0,0) \in E^{s} \times E^{s-1}$ such that for each $\left(u_{0}, \rho_{0}\right) \in U$ there exists a unique solution $(\varphi, f) \in C^{\infty}\left(J, H^{s} G_{0}\right)$ of (16) satisfying $(\varphi(0), f(0))=$ (id, 0$)$ and $\left(\varphi_{t}(0), f_{t}(0)\right)=\left(u_{0}, \rho_{0}\right)$. Furthermore, the solution depends smoothly on the initial data in the sense that the local flow $\Phi: J \times U \rightarrow H^{s} G_{0}$ defined by $\Phi\left(t, u_{0}, \rho_{0}\right)=\left(\varphi\left(t ; u_{0}, \rho_{0}\right), f\left(t ; u_{0}, \rho_{0}\right)\right)$ is a smooth map.

Writing the Cauchy problem for $2 \mathrm{HS}$ in the form

$$
\left\{\begin{array}{ccc}
u_{t}+u u_{x} & = & -\frac{1}{2} A^{-1}\left(u_{x}^{2}+\rho^{2}\right)_{x}, \\
\rho_{t}+u \rho_{x} & = & -\rho u_{x}, \\
(u(0), \rho(0)) & = & \left(u_{0}, \rho_{0}\right),
\end{array}\right.
$$

Theorem 4 implies that Eq. (17) in terms of the Euclidean variables $u=\varphi_{t} \circ \varphi^{-1}$ and $\rho=f_{t} \circ \varphi^{-1}$ is locally well-posed.

Corollary 5. Suppose $s>5 / 2$. Then for any $\left(u_{0}, \rho_{0}\right) \in E^{s} \times E^{s-1}$ there exists an open interval $J$ centered at 0 and a unique solution

$$
(u, \rho) \in C\left(J, E^{s} \times E^{s-1}\right) \cap C^{1}\left(J, E^{s-1} \times E^{s-2}\right)
$$

of the Cauchy problem (17) which depends continuously on the initial data $\left(u_{0}, \rho_{0}\right)$.

A similar result is true in the $C^{n}$-category with $n \geq 2$.

2.5. The $2 \mu \mathrm{HS}$ equation. We define a right-invariant metric on $H^{s} G, s>5 / 2$, which equals the inner product induced by $\mu-\partial_{x}^{2}$ for the first component plus the $L_{2}$ inner product for the second one at the identity, i.e.,

$$
\langle U, V\rangle_{(\varphi, f)}=\mu\left(u_{1} \circ \varphi^{-1}\right) \mu\left(v_{1} \circ \varphi^{-1}\right)+\int_{\mathbb{S}} \frac{u_{1 x} v_{1 x}}{\varphi_{x}} \mathrm{~d} x+\int_{\mathbb{S}} u_{2} v_{2} \varphi_{x} \mathrm{~d} x
$$

for any $U=\left(u_{1}, u_{2}\right), V=\left(v_{1}, v_{2}\right) \in T_{(\varphi, f)} H^{s} G$. With $\Gamma^{0}$ as in (11) we define a right-invariant Christoffel map for $2 \mu \mathrm{HS}$ by

$$
\Gamma_{(\mathrm{id}, 0)}(X, Y)=\left(\begin{array}{c}
\Gamma_{\mathrm{id}}^{0}\left(X_{1}, Y_{1}\right)-\frac{1}{2}\left(\mu-\partial_{x}^{2}\right)^{-1}\left(X_{2} Y_{2}\right)_{x} \\
-\frac{1}{2}\left(X_{1 x} Y_{2}+Y_{1 x} X_{2}\right)
\end{array}\right) .
$$

and an affine connection via (15). Then the following is easy to derive.

Proposition 6. Let $s>5 / 2$. Let $H^{s} G=H^{s} \operatorname{Diff}(\mathbb{S})\left(S H^{s-1}(\mathbb{S})\right.$ and let $\Gamma$ be the Christoffel map defined in (19). Then $\Gamma$ defines a smooth spray on $H^{s} G$, i.e., the map

$$
(\varphi, f) \mapsto \Gamma_{(\varphi, f)}: H^{s} G \rightarrow \mathcal{L}_{\text {sym }}^{2}\left(H^{s}(\mathbb{S}) \times H^{s-1}(\mathbb{S}) ; H^{s}(\mathbb{S}) \times H^{s-1}(\mathbb{S})\right)
$$


is smooth. Moreover, the metric $\langle\cdot, \cdot\rangle$ defined by (18) is a smooth (weak) Riemannian metric on $H^{s} G$, i.e., the map

$$
(\varphi, f) \mapsto\langle\cdot, \cdot\rangle_{(\varphi, f)}: H^{s} G \rightarrow \mathcal{L}_{\text {sym }}^{2}\left(T_{(\varphi, f)} H^{s} G ; \mathbb{R}\right)
$$

is a smooth section of the bundle $\mathcal{L}_{\mathrm{sym}}^{2}\left(T H^{s} G ; \mathbb{R}\right)$. Finally, the connection $\nabla$ is a Riemannian covariant derivative, compatible with $\langle\cdot, \cdot\rangle$.

We thus know that the $2 \mu \mathrm{HS}$ is a reexpression of the geodesic flow of the connection $\nabla$ defined in (15) on the product $H^{s} G$. The geodesic equation reads (16). We have the following local well-posedness result.

Theorem 7. Let $s>5 / 2$ and let $\Gamma$ be the $2 \mu H S$ Christoffel map. Then there exists an open interval $J$ centered at 0 and an open neighborhood $U$ of $(0,0) \in H^{s}(\mathbb{S}) \times$ $H^{s-1}(\mathbb{S})$ such that for each $\left(u_{0}, \rho_{0}\right) \in U$ there exists a unique solution $(\varphi, f) \in$ $C^{\infty}\left(J, H^{s} G\right)$ of (16) satisfying $(\varphi(0), f(0))=(\mathrm{id}, 0)$ and $\left(\varphi_{t}(0), f_{t}(0)\right)=\left(u_{0}, \rho_{0}\right)$. Furthermore, the solution depends smoothly on the initial data in the sense that the local flow $\Phi: J \times U \rightarrow H^{s} G$ defined by $\Phi\left(t, u_{0}, \rho_{0}\right)=\left(\varphi\left(t ; u_{0}, \rho_{0}\right), f\left(t ; u_{0}, \rho_{0}\right)\right)$ is a smooth map.

We write the Cauchy problem for $2 \mu \mathrm{HS}$ in the form

$$
\left\{\begin{array}{cccc}
u_{t}+u u_{x} & = & -\left(\mu-\partial_{x}^{2}\right)^{-1}\left(\frac{1}{2} u_{x}^{2}+2 \mu(u) u+\frac{1}{2} \rho^{2}\right)_{x}, \\
\rho_{t}+u \rho_{x} & = & -\rho u_{x}, \\
(u(0), \rho(0))= & \left(u_{0}, \rho_{0}\right) .
\end{array}\right.
$$

It follows from Theorem 7 that $2 \mu \mathrm{HS}$ is locally well-posed in $H^{s} \times H^{s-1}$ for $s>5 / 2$.

Corollary 8. Suppose $s>5 / 2$. Then for any $\left(u_{0}, \rho_{0}\right) \in H^{s}(\mathbb{S}) \times H^{s-1}(\mathbb{S})$ there exists an open interval $J$ centered at 0 and a unique solution

$$
(u, \rho) \in C\left(J, H^{s}(\mathbb{S}) \times H^{s-1}(\mathbb{S})\right) \cap C^{1}\left(J, H^{s-1}(\mathbb{S}) \times H^{s-2}(\mathbb{S})\right)
$$

of the Cauchy problem (20) which depends continuously on the initial data $\left(u_{0}, \rho_{0}\right)$.

The previous results hold with the obvious changes also in the $C^{n}$-category, $n \geq 2$.

\section{The Curvature of $H^{s} G_{0}$ Associated with the $2 \mathrm{HS}$}

Let us denote by

$$
R(X, Y) Z=\nabla_{X} \nabla_{Y} Z-\nabla_{Y} \nabla_{X} Z-\nabla_{[X, Y]} Z
$$

the curvature tensor of $H^{s} G_{0}$ equipped with the right-invariant metric (12). In the following theorem we compute an explicit formula for $R$ and show that the sectional curvature

$$
S(X, Y)=\frac{\langle R(X, Y) Y, X\rangle}{\langle X, X\rangle\langle Y, Y\rangle-\langle X, Y\rangle^{2}}
$$

has the constant positive value $1 / 4$.

Theorem 9. The curvature tensor for the $2 H S$ equation on $H^{s} G_{0}, s>5 / 2$, equipped with the right-invariant metric (12), for vector fields $X, Y, Z$, is given by

$$
4 R(X, Y) Z=X\langle Y, Z\rangle-Y\langle X, Z\rangle .
$$

In particular, the sectional curvature for $2 H S$ is constant and equal to $1 / 4$. 
Proof. We have the following local formula for $R$ in terms of the Christoffel map (14):

$R(X, Y) Z=D_{1} \Gamma_{p}(Z, X) Y-D_{1} \Gamma_{p}(Z, Y) X+\Gamma_{p}\left(\Gamma_{p}(Z, Y), X\right)-\Gamma_{p}\left(\Gamma_{p}(Z, X), Y\right)$, for any vector fields $X, Y, Z$ on $H^{s} G$, where $D_{1}$ denotes differentiation with respect to $p=(\varphi, f)$. By right-invariance of $\Gamma$, i.e.,

$$
\Gamma_{p}(X, Y) \circ \psi=\Gamma_{p \circ \psi}(X \circ \psi, Y \circ \psi),
$$

it holds that

$$
R(X, Y) Z \circ \varphi^{-1}=R(u, v) w
$$

if $X=u \circ \varphi, Y=v \circ \varphi$ and $Z=w \circ \varphi$. Therefore, it suffices to consider the curvature at (id, 0$)$. We write $\Gamma=\Gamma_{(\mathrm{id}, 0)}$ and denote the components of $u$ by $u_{1}$ and $u_{2}$ and similarly for $v, w$. A lengthy but straightforward computation shows that

$$
\begin{aligned}
R(u, v) w= & D_{1} \Gamma(w, u) v-D_{1} \Gamma(w, v) u+\Gamma(\Gamma(w, v), u)-\Gamma(\Gamma(w, u), v), \\
=\quad- & \Gamma\left(w_{x} v_{1}, u\right)-\Gamma\left(u_{x} v_{1}, w\right)+\Gamma(w, u)_{x} v_{1} \\
& +\Gamma\left(w_{x} u_{1}, v\right)+\Gamma\left(v_{x} u_{1}, w\right)-\Gamma(w, v)_{x} u_{1} \\
& +\Gamma(\Gamma(w, v), u)-\Gamma(\Gamma(w, u), v) .
\end{aligned}
$$

In the first component, we have the terms

$$
\begin{aligned}
& -\Gamma^{0}\left(w_{1 x} v_{1}, u_{1}\right)+\frac{1}{2} A^{-1}\left(w_{2 x} v_{1} u_{2}\right)_{x}-\Gamma^{0}\left(u_{1 x} v_{1}, w_{1}\right)+\frac{1}{2} A^{-1}\left(u_{2 x} v_{1} w_{2}\right)_{x} \\
& +\Gamma^{0}\left(w_{1}, u_{1}\right)_{x} v_{1}-\frac{1}{2}\left[A^{-1}\left(w_{2} u_{2}\right)_{x}\right]_{x} v_{1}+\Gamma^{0}\left(w_{1 x} u_{1}, v_{1}\right)-\frac{1}{2} A^{-1}\left(w_{2 x} u_{1} v_{2}\right)_{x} \\
& +\Gamma^{0}\left(v_{1 x} u_{1}, w_{1}\right)-\frac{1}{2} A^{-1}\left(v_{2 x} u_{1} w_{2}\right)_{x}-\Gamma^{0}\left(w_{1}, v_{1}\right)_{x} u_{1}+\frac{1}{2}\left[A^{-1}\left(w_{2} v_{2}\right)_{x}\right]_{x} u_{1} \\
& +\Gamma^{0}\left(\Gamma^{0}\left(w_{1}, v_{1}\right)-\frac{1}{2} A^{-1}\left(w_{2} v_{2}\right)_{x}, u_{1}\right)+\frac{1}{4} A^{-1}\left(w_{1 x} v_{2} u_{2}+v_{1 x} w_{2} u_{2}\right)_{x} \\
& -\Gamma^{0}\left(\Gamma^{0}\left(w_{1}, u_{1}\right)-\frac{1}{2} A^{-1}\left(w_{2} u_{2}\right)_{x}, v_{1}\right)-\frac{1}{4} A^{-1}\left(w_{1 x} u_{2} v_{2}+u_{1 x} w_{2} v_{2}\right)_{x} .
\end{aligned}
$$

Using that

$$
\partial_{x} A^{-1} \partial_{x}=\mu-1
$$

and the relation

$$
\Gamma^{0}\left(\Gamma^{0}\left(w_{1}, v_{1}\right), u_{1}\right)-\Gamma^{0}\left(\Gamma^{0}\left(w_{1}, u_{1}\right), v_{1}\right)=-\frac{1}{4} u_{1} \mu\left(w_{1 x} v_{1 x}\right)+\frac{1}{4} v_{1} \mu\left(w_{1 x} u_{1 x}\right),
$$

cf. 23], we see that these terms equal

$$
\begin{aligned}
& \frac{1}{2} A^{-1} \partial_{x}\left[\left(w_{1 x} v_{1}\right)_{x} u_{1 x}+\left(u_{1 x} v_{1}\right)_{x} w_{1 x}-\left(w_{1 x} u_{1}\right)_{x} v_{1 x}-\left(v_{1 x} u_{1}\right)_{x} w_{1 x}\right] \\
& -\frac{1}{2}(\mu-1)\left(w_{1 x} u_{1 x}\right) v_{1}+\frac{1}{2}(\mu-1)\left(w_{1 x} v_{1 x}\right) u_{1} \\
& +\frac{1}{2} A^{-1}\left[\left(w_{2 x} v_{1} u_{2}\right)_{x}+\left(u_{2 x} v_{1} w_{2}\right)_{x}-\left(w_{2 x} u_{1} v_{2}\right)_{x}-\left(v_{2 x} u_{1} w_{2}\right)_{x}\right] \\
& -\frac{1}{2}(\mu-1)\left(w_{2} u_{2}\right) v_{1}+\frac{1}{2}(\mu-1)\left(w_{2} v_{2}\right) u_{1} \\
& -\frac{1}{4} u_{1} \mu\left(w_{1 x} v_{1 x}\right)+\frac{1}{4} v_{1} \mu\left(w_{1 x} u_{1 x}\right)-\frac{1}{2} A^{-1} \partial_{x}\left[\left(-\frac{1}{2}(\mu-1)\left(w_{2} v_{2}\right)\right) u_{1 x}\right] \\
(21) \quad & +\frac{1}{2} A^{-1} \partial_{x}\left[\left(-\frac{1}{2}(\mu-1)\left(w_{2} u_{2}\right)\right) v_{1 x}\right]+\frac{1}{4} A^{-1} \partial_{x}\left(v_{1 x} w_{2} u_{2}-u_{1 x} w_{2} v_{2}\right) .
\end{aligned}
$$


To see that the terms with $A^{-1} \partial_{x}$ cancel out, we use that $u_{1}(0)=v_{1}(0)=0$ so that

$$
\begin{aligned}
\frac{1}{2} w_{1 x} u_{1 x} v_{1}-\frac{1}{2} u_{1} w_{1 x} v_{1 x} & =-A^{-1} \partial_{x}^{2}\left(\frac{1}{2} w_{1 x} u_{1 x} v_{1}-\frac{1}{2} u_{1} w_{1 x} v_{1 x}\right) \\
& =\frac{1}{2} A^{-1} \partial_{x}\left(w_{1 x x}\left(u_{1} v_{1 x}-u_{1 x} v_{1}\right)\right. \\
& \left.+w_{1 x} u_{1} v_{1 x x}-w_{1 x} u_{1 x x} v_{1}\right),
\end{aligned}
$$

which coincides up to sign with the first row terms in (21), and

$$
\begin{aligned}
\frac{1}{2} w_{2} u_{2} v_{1}-\frac{1}{2} w_{2} v_{2} u_{1}= & -A^{-1} \partial_{x}^{2}\left(\frac{1}{2} w_{2} u_{2} v_{1}-\frac{1}{2} w_{2} v_{2} u_{1}\right) \\
= & \frac{1}{2} A^{-1} \partial_{x}\left(w_{2 x}\left(v_{2} u_{1}-u_{2} v_{1}\right)+w_{2}\left(v_{2 x} u_{1}-u_{2 x} v_{1}\right)\right. \\
& \left.\quad+w_{2}\left(v_{2} u_{1 x}-u_{2} v_{1 x}\right)\right) .
\end{aligned}
$$

Using $A^{-1} \partial_{x}^{2} v_{1}=-v_{1}$ and $A^{-1} \partial_{x}^{2} u_{1}=-u_{1}$, the first component terms (21) thus reduce to

$$
\frac{1}{4} u_{1}\left(\mu\left(w_{1 x} v_{1 x}\right)+\mu\left(w_{2} v_{2}\right)\right)-\frac{1}{4} v_{1}\left(\mu\left(w_{1 x} u_{1 x}\right)+\mu\left(w_{2} u_{2}\right)\right),
$$

which is the desired expression. The second component terms are

$$
\begin{aligned}
& \frac{1}{2}\left[\left(w_{1 x} v_{1}\right)_{x} u_{2}+u_{1 x} w_{2 x} v_{1}\right]+\frac{1}{2}\left[\left(u_{1 x} v_{1}\right)_{x} w_{2}+w_{1 x} u_{2 x} v_{1}\right]-\frac{1}{2} v_{1}\left[w_{1 x} u_{2}+u_{1 x} w_{2}\right]_{x} \\
& -\frac{1}{2}\left[\left(w_{1 x} u_{1}\right)_{x} v_{2}+v_{1 x} w_{2 x} u_{1}\right]-\frac{1}{2}\left[\left(v_{1 x} u_{1}\right)_{x} w_{2}+w_{1 x} v_{2 x} u_{1}\right]+\frac{1}{2} u_{1}\left[w_{1 x} v_{2}+v_{1 x} w_{2}\right]_{x} \\
& -\frac{1}{2}\left(\Gamma_{1}(w, v)_{x} u_{2}+u_{1 x} \Gamma_{2}(w, v)\right)+\frac{1}{2}\left(\Gamma_{1}(w, u)_{x} v_{2}+v_{1 x} \Gamma_{2}(w, u)\right)
\end{aligned}
$$

and with $\partial_{x} A^{-1} \partial_{x}=\mu-1$ we can simplify the last row terms

$$
\Gamma_{1}(w, v)_{x}=\frac{1}{2} w_{1 x} v_{1 x}-\frac{1}{2} \mu\left(w_{1 x} v_{1 x}\right)+\frac{1}{2} w_{2} v_{2}-\frac{1}{2} \mu\left(w_{2} v_{2}\right)
$$

and

$$
\Gamma_{1}(w, u)_{x}=\frac{1}{2} w_{1 x} u_{1 x}-\frac{1}{2} \mu\left(w_{1 x} u_{1 x}\right)+\frac{1}{2} w_{2} u_{2}-\frac{1}{2} \mu\left(w_{2} u_{2}\right) .
$$

It is now easy to see that the terms in (22) reduce to

$$
\frac{1}{4} u_{2}\left(\mu\left(w_{1 x} v_{1 x}\right)+\mu\left(w_{2} v_{2}\right)\right)-\frac{1}{4} v_{2}\left(\mu\left(w_{1 x} u_{1 x}\right)+\mu\left(w_{2} u_{2}\right)\right)
$$

so that we obtain

$$
R(u, v) w=\frac{1}{4} u\langle v, w\rangle-\frac{1}{4} v\langle u, w\rangle .
$$

By the definition of the sectional curvature, we have

$$
S(u, v)=\frac{\langle R(u, v) v, u\rangle}{\langle u, u\rangle\langle v, v\rangle-\langle u, v\rangle^{2}}=\frac{1}{4} .
$$

Remark 10. Since Lenells [23] uses a different scaling for the $\dot{H}^{1}$-metric, he comes to the result that the sectional curvature for the HS is identically equal to 1 . Note carefully that we have only used that $u_{1}$ and $v_{1}$ vanish at zero; a corresponding assumption on the second components is not necessary in the above proof. 
The above result is of particular interest concerning the stability of the geodesic flow associated with the $2 \mathrm{HS}$ system, cf. the appendix. The very recent paper 36] explains that the geodesic flow for the 2-component Hunter-Saxton system allows a continuation (beyond the breaking time of the associated solution $(u, \rho)$ of the original equation) on the space $\mathcal{M}_{A C}^{0}=M^{A C}\left(\mathrm{~S} H^{0}(\mathbb{S})\right.$, where $M^{A C}$ is the set of nondecreasing absolutely continuous functions $\varphi:[0,1] \rightarrow[0,1]$ with $\varphi(0)=0$ and $\varphi(1)=1$. Precisely, the flow variables $(\varphi, f) \in \mathcal{M}_{A C}^{0}$ for 2 HS satisfy the geodesic equation $\left(\varphi_{t t}, f_{t t}\right)=\Gamma_{(\varphi, f)}\left(\left(\varphi_{t}, f_{t}\right),\left(\varphi_{t}, f_{t}\right)\right)$ for any positive $t$. Interestingly, the space $M^{A C}$ is bijective to the open subset

$$
\mathcal{U}^{0}=\left\{f \in H^{0}(\mathbb{S}) ;\|f\|_{H^{0}}=1, f>0 \text { a.e. on } \mathbb{S}\right\}
$$

of the $L_{2}$ unit sphere via the mapping

$$
f \mapsto\left(x \mapsto \varphi(x)=\int_{0}^{x} f^{2}(y) \mathrm{d} y\right) .
$$

Lenells [23, 24] used the constance and positivity of the sectional curvature associated with HS as a motivation to continue the geodesic flow on the infinitedimensional sphere $M^{A C}$, keeping in mind the well-known fact that any finite dimensional Riemannian manifold with constant positive curvature is locally isometric to a sphere. In the interim, Wunsch [36] already adopted this approach for the $2 \mathrm{HS}$ system so that our work may serve retroactively as a motivation for considering the problem of extending the flow variables on a suitable configuration space (which in fact works by multiplying the sphere $M^{A C}$ with $H^{0}(\mathbb{S})$ ).

\section{The curvature of $H^{s} G$ associated with the $2 \mu \mathrm{HS}$}

In this section, $R$ and $S$ stand for the curvature tensor and the unnormalized sectional curvature for the $2 \mu \mathrm{HS}$ equation.

Theorem 11. The unnormalized sectional curvature $S(u, v)$ for the $2 \mu H S$ equation is given by

$$
S(u, v)=\langle\Gamma(u, v), \Gamma(u, v)\rangle-\langle\Gamma(u, u), \Gamma(v, v)\rangle-3 \mu\left(u_{1 x} v_{1}\right)^{2} .
$$

Proof. The proof is similar to the proof of Proposition 5.1 in [11]; the single difference is that the additional terms involving $\Gamma^{0}$ in (5.4) of 11] do not cancel out but give $-3 \mu\left(u_{1 x} v_{1}\right)^{2}$ as explained in [20].

In the following we write $S_{1}, S_{2}$ to distinguish between the sectional curvature for the one-component $\mu \mathrm{HS}$ and its two-component extension. In [20], the authors prove that $S_{1}\left(u_{1}, v_{1}\right)$ is always positive for any two orthonormal vectors $u_{1}$ and $u_{2}$ (with respect to the scalar product induced by $\mu-\partial_{x}^{2}$ ). Since we have

$$
S_{2}\left(\left(\begin{array}{c}
u_{1} \\
0
\end{array}\right),\left(\begin{array}{c}
v_{1} \\
0
\end{array}\right)\right)=S_{1}\left(u_{1}, v_{1}\right)
$$

we see that $S_{2}$ is positive in the $H^{s} \operatorname{Diff}(\mathbb{S})$-direction. To find a large subspace of positive sectional curvature for $2 \mu \mathrm{HS}$ with non-trival second component we compute $S_{2}(u, v)$ for

$$
u=\left(\begin{array}{c}
\cos k_{1} x \\
\cos k_{2} x
\end{array}\right), \quad v=\left(\begin{array}{c}
\cos l_{1} x \\
\cos l_{2} x
\end{array}\right)
$$


where $k_{i} \neq l_{i} \in 2 \pi \mathbb{N}, i=1,2$; this is inspired by calculations in [1] and 26] where the authors show that the sectional curvature for $\mathrm{CH}$ is positive for any pair of trigonometric functions. Note that

$$
\begin{aligned}
S_{2}(u, v)= & S_{1}\left(u_{1}, v_{1}\right)+\frac{1}{4} \int_{\mathbb{S}}\left(u_{2} v_{2}\right)_{x}\left(\mu-\partial_{x}^{2}\right)^{-1}\left(u_{2} v_{2}\right)_{x} \mathrm{~d} x-\int_{\mathbb{S}} \Gamma^{0}\left(u_{1}, v_{1}\right)\left(u_{2} v_{2}\right)_{x} \mathrm{~d} x \\
& +\frac{1}{4} \int_{\mathbb{S}}\left(u_{1 x} v_{2}+v_{1 x} u_{2}\right)^{2} \mathrm{~d} x-\frac{1}{4} \int_{\mathbb{S}}\left(u_{2}^{2}\right)_{x}\left(\mu-\partial_{x}^{2}\right)^{-1}\left(v_{2}^{2}\right)_{x} \mathrm{~d} x \\
& +\frac{1}{2} \int_{\mathbb{S}} \Gamma^{0}\left(u_{1}, u_{1}\right)\left(v_{2}^{2}\right)_{x} \mathrm{~d} x+\frac{1}{2} \int_{\mathbb{S}} \Gamma^{0}\left(v_{1}, v_{1}\right)\left(u_{2}^{2}\right)_{x} \mathrm{~d} x-\int_{\mathbb{S}} u_{1 x} u_{2} v_{1 x} v_{2} \mathrm{~d} x \\
= & S_{1}\left(u_{1}, v_{1}\right)+\sum_{j=1}^{4} I_{j},
\end{aligned}
$$

where

$$
\begin{aligned}
I_{1}= & \frac{1}{4} \int_{\mathbb{S}}\left(u_{2} v_{2}\right)_{x}\left(\mu-\partial_{x}^{2}\right)^{-1}\left(u_{2} v_{2}\right)_{x} \mathrm{~d} x \\
I_{2}= & -\frac{1}{4} \int_{\mathbb{S}}\left(u_{2}^{2}\right)_{x}\left(\mu-\partial_{x}^{2}\right)^{-1}\left(v_{2}^{2}\right)_{x} \mathrm{~d} x \\
I_{3}= & -\int_{\mathbb{S}} \Gamma^{0}\left(u_{1}, v_{1}\right)\left(u_{2} v_{2}\right)_{x} \mathrm{~d} x+\frac{1}{2} \int_{\mathbb{S}} \Gamma^{0}\left(u_{1}, u_{1}\right)\left(v_{2}^{2}\right)_{x} \mathrm{~d} x \\
& \quad+\frac{1}{2} \int_{\mathbb{S}} \Gamma^{0}\left(v_{1}, v_{1}\right)\left(u_{2}^{2}\right)_{x} \mathrm{~d} x, \\
I_{4}= & \frac{1}{4} \int_{\mathbb{S}}\left(u_{1 x} v_{2}+v_{1 x} u_{2}\right)^{2} \mathrm{~d} x-\int_{\mathbb{S}} u_{1 x} u_{2} v_{1 x} v_{2} \mathrm{~d} x .
\end{aligned}
$$

We write $A=\mu-\partial_{x}^{2}$ and apply the identity

$$
A^{-1} \partial_{x}^{2}=\partial_{x} A^{-1} \partial_{x}=\partial_{x}^{2} A^{-1}=\mu-1 .
$$

Using integration by parts and the orthogonality relations for trigonometric functions we find

$$
\begin{aligned}
S_{1}\left(u_{1}, v_{1}\right)= & \left\langle\Gamma^{0}\left(u_{1}, v_{1}\right), \Gamma^{0}\left(u_{1}, v_{1}\right)\right\rangle-\left\langle\Gamma^{0}\left(u_{1}, u_{1}\right), \Gamma^{0}\left(v_{1}, v_{1}\right)\right\rangle-3 \mu\left(u_{1 x} v_{1}\right)^{2} \\
= & -\frac{1}{2} \int_{\mathbb{S}} A^{-1}\left[\partial_{x}\left(u_{1 x} v_{1 x}\right)\right] A \Gamma^{0}\left(u_{1}, v_{1}\right) \mathrm{d} x \\
& \quad+\frac{1}{2} \int_{\mathbb{S}} A^{-1}\left[\partial_{x}\left(u_{1 x}^{2}\right)\right] A \Gamma^{0}\left(v_{1}, v_{1}\right) \mathrm{d} x \\
= & \frac{1}{2} \int_{\mathbb{S}} u_{1 x} v_{1 x} \Gamma^{0}\left(u_{1}, v_{1}\right)_{x} \mathrm{~d} x-\frac{1}{2} \int_{\mathbb{S}} u_{1 x}^{2} \Gamma^{0}\left(v_{1}, v_{1}\right)_{x} \mathrm{~d} x \\
= & -\frac{1}{4} \int_{\mathbb{S}} u_{1 x} v_{1 x}\left(A^{-1} \partial_{x}^{2}\right)\left(u_{1 x} v_{1 x}\right) \mathrm{d} x+\frac{1}{4} \int_{\mathbb{S}} u_{1 x}^{2}\left(A^{-1} \partial_{x}^{2}\right)\left(v_{1 x}^{2}\right) \mathrm{d} x \\
= & \frac{1}{4} \mu\left(u_{1 x}^{2}\right) \mu\left(v_{1 x}^{2}\right) \\
= & \frac{1}{16} k_{1}^{2} l_{1}^{2} .
\end{aligned}
$$

Our choice of $k_{1}$ and $l_{1}$ implies that the one-component sectional curvature is strictly positive. All we have to show is that the second component terms do not contribute 
negative terms which make the total sectional curvature negative. Similar computations show that the terms $I_{1}$ and $I_{2}$ in (23) are

$$
I_{1}=-\frac{1}{4} \int_{\mathbb{S}} u_{2} v_{2} \partial_{x}^{2} A^{-1} u_{2} v_{2} \mathrm{~d} x=\frac{1}{4} \int_{\mathbb{S}} u_{2} v_{2}(1-\mu)\left(u_{2} v_{2}\right) \mathrm{d} x=\frac{1}{4} \int_{\mathbb{S}} u_{2}^{2} v_{2}^{2} \mathrm{~d} x
$$

and

$$
I_{2}=\frac{1}{4} \int_{\mathbb{S}} u_{2}^{2} \partial_{x}^{2} A^{-1} v_{2}^{2} \mathrm{~d} x=\frac{1}{4} \int_{\mathbb{S}} u_{2}^{2}(\mu-1) v_{2}^{2} \mathrm{~d} x=-\frac{1}{4} \int_{\mathbb{S}} u_{2}^{2} v_{2}^{2} \mathrm{~d} x+\frac{1}{16} .
$$

Since

$$
\begin{aligned}
-\int_{\mathbb{S}} \Gamma^{0}\left(u_{1}, v_{1}\right)\left(u_{2} v_{2}\right)_{x} \mathrm{~d} x & =\frac{1}{2} \int_{\mathbb{S}} A^{-1}\left(u_{1 x} v_{1 x}\right)_{x}\left(u_{2} v_{2}\right)_{x} \mathrm{~d} x \\
& =\frac{1}{2} \int_{\mathbb{S}}\left[(1-\mu)\left(u_{1 x} v_{1 x}\right)\right] u_{2} v_{2} \mathrm{~d} x \\
& =\frac{1}{2} \int_{\mathbb{S}} u_{1 x} u_{2} v_{1 x} v_{2} \mathrm{~d} x
\end{aligned}
$$

we find that

$$
\begin{aligned}
I_{3}+I_{4}= & \frac{1}{2} \int_{\mathbb{S}} \Gamma^{0}\left(u_{1}, u_{1}\right)\left(v_{2}^{2}\right)_{x} \mathrm{~d} x+\frac{1}{2} \int_{\mathbb{S}} \Gamma^{0}\left(v_{1}, v_{1}\right)\left(u_{2}^{2}\right)_{x} \mathrm{~d} x \\
& \quad+\frac{1}{4} \int_{\mathbb{S}}\left(u_{1 x}^{2} v_{2}^{2}+v_{1 x}^{2} u_{2}^{2}\right) \mathrm{d} x \\
= & \frac{1}{4} \mu\left(u_{1 x}^{2}\right) \mu\left(v_{2}^{2}\right)+\frac{1}{4} \mu\left(v_{1 x}^{2}\right) \mu\left(u_{2}^{2}\right) \\
= & \frac{1}{16}\left(k_{1}^{2}+l_{1}^{2}\right) .
\end{aligned}
$$

It follows from (23) and (24) that

$$
S_{2}(u, v)=\frac{1}{16}\left(1+k_{1}^{2}+l_{1}^{2}+k_{1}^{2} l_{1}^{2}\right)>\frac{1}{16} .
$$

Our calculation also shows that the sectional curvature is equal to $1 / 16$ in the direction of the second component since

$$
S_{2}\left(\left(\begin{array}{c}
0 \\
u_{2}
\end{array}\right),\left(\begin{array}{c}
0 \\
v_{2}
\end{array}\right)\right)=I_{1}+I_{2}=\frac{1}{16} .
$$

We have thus shown the following proposition.

Proposition 12. Let $s>5 / 2$. Let $S(u, v):=\langle R(u, v) v, u\rangle$ be the unnormalized sectional curvature on $H^{s} G$ associated with the $2 \mu H S$ equation. Then

$$
S(u, v)>\frac{1}{16}
$$

for all vectors $u, v \in T_{(\mathrm{id}, 0)} H^{s} G$, of the form

$$
u=\left(\begin{array}{c}
\cos k_{1} x \\
\cos k_{2} x
\end{array}\right), \quad v=\left(\begin{array}{c}
\cos l_{1} x \\
\cos l_{2} x
\end{array}\right), \quad k_{i} \neq l_{i} \in\{2 \pi, 4 \pi, \ldots\} .
$$

Moreover, the normalized sectional curvature satisfies

$$
\frac{S(u, v)}{\langle u, u\rangle\langle v, v\rangle-\langle u, v\rangle^{2}}=\frac{1}{4}
$$

for all vectors $u, v \in T_{(\mathrm{id}, 0)} H^{s} G$ of the form

$$
u=\left(\begin{array}{c}
0 \\
\cos k_{2} x
\end{array}\right), \quad v=\left(\begin{array}{c}
0 \\
\cos l_{2} x
\end{array}\right), \quad k_{2} \neq l_{2} \in\{2 \pi, 4 \pi, \ldots\} .
$$


It is explained in 20] that the first-component configuration space $H^{s} \operatorname{Diff}(\mathbb{S})$ can be thought of as a solid torus with a cross-section isomorphic to $H^{s} \operatorname{Diff}(\mathbb{S}) / \mathbb{S}$. The fact that the one-component sectional curvature is positive is proved by a decomposition of the tangent space $T_{\mathrm{id}} H^{s} \operatorname{Diff}(\mathbb{S})=U \oplus V$ corresponding to the decomposition $u=\tilde{u}+\mu(u)$, where $\mu(\tilde{u})=0$, i.e., $U$ are the zero mean functions and $V \simeq \mathbb{R}$ are the constants. It is an open problem and a task for further research which geometric interpretations for the group $H^{s} G$ associated with the $2 \mu \mathrm{HS}$ system can be given and which conclusions for the (non)existence of solutions of $2 \mu \mathrm{HS}$ can be drawn. While the present section shows the existence of subspaces of positive sectional curvature for $2 \mu \mathrm{HS}$, one could ask whether $S_{2}$ is always positive, for arbitrary second component functions, or whether there are directions of strictly negative sectional curvature.

\section{Appendix: Generalities on GeOdesic flows on infinite dimensional LIE GROUPS AND THEIR STABILITY PROPERTIES}

In this appendix, we will survey the most important results of the seminal papers [1, 10] which are relevant for the purposes of the paper at hand. It goes back to Arnold's work [1] to model both Euler's equation for a rotating rigid body and Euler's equation for an ideal fluid on a Lie group with an invariant metric. The Lie group for the rotating rigid body is the matrix group $S O(3)$, whereas the motion of an ideal fluid is modeled on the diffeomorphism group $\operatorname{Diff}(M)$ of volume preserving diffeomorphisms of a certain manifold $M$. While the matrix group $S O(3)$ has finite dimension and is equipped with a left-invariant metric, the group $\operatorname{Diff}(M)$ is an infinite dimensional Lie group which is equipped with a right-invariant metric. The geometric viewpoint is not only aesthetically appealing, but is also very useful for the study of well-posedness and stability issues. In view of the results of the present paper, we provide a general overview about the geometric picture for ideal fluids (which corresponds to a right-invariant formulation), with a focus on the stability of the geodesic flow.

Let $G$ be a (not necessarily finite dimensional) Lie group with Lie algebra $\mathfrak{g} \simeq$ $T_{e} G$, where $e$ denotes the unit element. We assume that there is an invertible linear operator $A: \mathfrak{g} \rightarrow \mathfrak{g}^{*}$ which is, for historical reasons, going back to Euler's work on the rigid body motion, called an inertia operator. We also assume that $\mathfrak{g}^{*} \simeq \mathfrak{g}$ (which can often be achieved by considering a suitable subspace of $\mathfrak{g}^{*}$ ) so that $A$ can in fact be regarded as an automorphism of $\mathfrak{g}$. Let $R_{g}: G \rightarrow G$ denote the right translation map on $G$. We obtain a right-invariant metric $\rho_{A}$ on $G$ by setting

$$
\rho_{A}(u, v)=\left(A\left[D R_{g^{-1}} u\right], D R_{g^{-1}} v\right)
$$

for all $u, v \in T_{g} G$, where $(\cdot, \cdot)$ denotes the dual pairing on $\mathfrak{g}^{*} \times \mathfrak{g}$. If $G$ is infinite dimensional, the map $\rho_{A}$ defines in general only a weak Riemannian metric on $G$, i.e., the natural topology on any tangent space $T_{g} G$ is stronger than the topology induced by the metric $\rho_{A}$, cf. [10]. Let ad ${ }_{u}^{*}$ denote the dual operator (with respect to $\rho_{A}$ ) of the natural action of the Lie algebra on itself given by $\operatorname{ad}_{u}: \mathfrak{g} \rightarrow \mathfrak{g}, v \mapsto[u, v]$, and define the bilinear and symmetric map

$$
B_{e}: \mathfrak{g} \times \mathfrak{g} \rightarrow \mathfrak{g}, \quad B_{e}(u, v)=\frac{1}{2}\left(\operatorname{ad}_{u}^{*} v+\operatorname{ad}_{v}^{*} u\right) .
$$


Next, we introduce an affine connection on $G$ given by

$$
\nabla_{\xi_{u}} \xi_{v}=\frac{1}{2}\left[\xi_{u}, \xi_{v}\right]+B\left(\xi_{u}, \xi_{v}\right)
$$

where $\xi_{u}$ is the right-invariant vector field on $G$ with value $u$ at $e$ and $B$ denotes the right-invariant tensor field with value $B_{e}$ at the identity. Let $g(t)$ be a smooth part in $G$ and define its Eulerian velocity, which lies in the Lie algebra $\mathfrak{g}$, by

$$
u(t)=D R_{g^{-1}(t)} \dot{g}(t) .
$$

The crucial point is that $g(t)$ is a geodesic for the connection $\nabla$ if and only if its Eulerian velocity satisfies the Euler equation

$$
u_{t}=-B(u, u)
$$

see [12] for instance.

Interestingly the above formalism also works the other way round: Starting from an Euler equation $u_{t}=-B(u, u)$ with quadratic right-hand side, defined on the Lie algebra $\mathfrak{g}$ of some Lie group $G$, and defining the connection $\nabla$ in terms of the operator $B$ as in (25), one sees that the Euler equation re-expresses a geodesic flow on the Lie group $G$. Nevertheless, it is not clear that there is a right-invariant metric $\rho_{A}$ on $G$, induced by some inertia operator $A$, such that the connection $\nabla$ is compatible with the metric in the sense that

$$
X\left(\rho_{A}(Y, Z)\right)=\rho_{A}\left(\nabla_{X} Y, Z\right)+\rho_{A}\left(\nabla_{X} Z, Y\right),
$$

for vector fields $X, Y, Z$ on $G$. Recall that the equations under discussion in the main body of this paper are metric in the sense that they allow for a Riemannian structure.

Let $x(t)$ denote a geodesic in $G$ and consider the geodesic variation $x(t, s)$ with the associated variation vector field

$$
\left.\frac{\mathrm{d} x(t, s)}{\mathrm{d} s}\right|_{s=0}=\xi(t) \in T_{x(t)} G .
$$

It is well-known that $\xi$ is a solution of the Jacobi equation

$$
\frac{D^{2} \xi}{D t^{2}}=-R(\xi, v) v
$$

where $D / D t$ is the covariant derivative, $R$ is the curvature tensor associated with the connection $\nabla$ and $v=\dot{x}(t)$ is the velocity field. By a decomposition of the variation vector $\xi$ into components parallel and perpendicular to the velocity $v$, Arnold showed that the Jacobi equation for the perpendicular component (which is henceforth also denoted as $\xi$ for simplicity) can be written in the form

$$
\frac{D^{2} \xi}{D t^{2}}=-\operatorname{grad} U, \quad U=\frac{S}{2} \rho_{A}(\xi, \xi) \rho_{A}(v, v)
$$

here $S$ is the sectional curvature of the two-dimensional subspace of $T_{x(t)} G$ spanned by $v$ and $\xi$. If the norm of $v$ is equal to 1 (which can be achieved by a parametrization of the geodesic by arc length), the Jacobi equation for $\xi$ reduces to the harmonic oscillator equation with the potential energy $U$ equal to the product of the curvature in the direction spanned by the velocity vector and the normal component of the variation with the square of length of this normal component. From this, it is obvious that $S<0$ implies an exponential divergence of the geodesics starting near $x(0)$, whereas for $S>0$, convergence of the nearby geodesis is expected; cf. [2] for further details. This motivates our research for subspaces of positive sectional 
curvature in the main body of the paper. Note that curvature computations for geometric evolution equations have a long tradition: They have already been carried out in the 1980's 14, 29] and in Misiołek's paper [30] about the Camassa-Holm equation; see also 22] for a more recent paper.

Acknowledgements. The author thanks Jonatan Lenells (Baylor University, Waco) and Joachim Escher (Leibniz University, Hannover) for bringing the above problems to his attention. A cordial thank for useful remarks that helped to improve the manuscript goes to the anonymous referees.

\section{REFERENCES}

[1] Arnold, V.I.: Sur la géométrie différentielle des groupes de Lie de dimension infinie et ses applications à l'hydrodynamique des fluides parfaits. Ann. Inst. Fourier (Grenoble) 16, 319-361 (1966)

[2] Arnold, V.I.: Mathematical Methods of Classical Mechanics. GTM 60, Springer, New York 1989.

[3] Beals, R., Sattinger, D.H., Szmigielski, J.: Inverse scattering solutions of the Hunter-Saxton equation. Appl. Anal. 78, no. 3\&4, 255-269 (2001)

[4] Camassa, R., Holm, D.D.: An integrable shallow water equation with peaked solitons. Phys. Rev. Lett. 71, no. 11, 1661-1664 (1993)

[5] Chae, D.: On the blow-up problem for the axisymmetric 3D Euler equations. Nonlinearity 21, 2053-2060 (2008)

[6] Constantin, A.: Global solutions of the Hunter-Saxton equation. SIAM J. Math. Anal. 37(3), 996-1026 (2005)

[7] Constantin, A., Ivanov, R.: On an integrable two-component Camassa-Holm shallow water system. Phys. Lett. A 372, 7129-7132 (2008)

[8] Constantin, A., Lax, P., Majda, A.: A simple one-dimensional model for the three-dimensional vorticity equation. Commun. Pure Appl. Math. 38, 715-724 (1985)

[9] Dai, H.H., Pavlov, M.: Transformations for the Camassa-Holm equation, its high-frequency limit and the Sinh-Gordon equation. J. Phys. Soc. Japan 67 (1998), 3655-3657

[10] Ebin, D.G., Marsden, J.: Groups of diffeomorphisms and the motion of an incompressible fluid. Ann. of Math. 92, no. 2, 102-163 (1970)

[11] Escher, J., Kohlmann, M., Lenells, J.: The geometry of the two-component Camassa-Holm and Degasperis-Procesi equations. J. Geom. Phys. 61 (2011) $436-452$

[12] Escher, J., Kolev, B.: The Degasperis-Procesi equation as a non-metric Euler equation. Math. Z., DOI 10.1007/s00209-010-0778-2

[13] Escher, J., Lechtenfeld, O., Yin, Z.: Well-posedness and blow-up phenomena for the 2-component Camassa-Holm equation. DCDS 19, 493-513 (2007)

[14] Freed, D.: The geometry of loop groups. J. Differential Geom. 28, 223-276 (1988)

[15] Holm, D.D., Marsden, J.E., Ratiu, T.S.: The Euler-Poincaré equations and semidirect products with applications to continuum theories. Adv. in Math. 137, 1-81 (1998) 
[16] Holm, D.D., Tronci, C.: Geodesic flows on semidirect-product Lie groups: geometry of singular measure-valued solutions. Proc. R. Soc. A 465, 457-476 (2009)

[17] Hou, T.Y., Li. C.: Dynamic stability of the three-dimensional axisymmetric Navier-Stokes equations with swirl. Comm. Pure Appl. Math. LXI, 661-697 (2008)

[18] Hunter, J.K., Saxton, R.: Dynamics of director fields. SIAM J. Appl. Math. 51, 1498-1521 (1991)

[19] Hunter, J.K., Zheng, Y.: On a completely integrable nonlinear hyperbolic variational equation. Physica D 79 (1994), 361-386

[20] Khesin, B., Lenells, J., Misiołek, G.: Generalized Hunter-Saxton equation and the geometry of the group of circle diffeomorphisms. Math. Ann. 342, 617-656 (2008)

[21] Lechtenfeld, O., Lenells, J.: On the $N=2$ supersymmetric Camassa-Holm and Hunter-Saxton equations. J. Math. Phys. 50 (2009) 012704

[22] Lenells, J.: Riemannian geometry on the diffeomorphism group of the circle. Ark. Mat. 45, 297-325 (2007)

[23] Lenells, J.: The Hunter-Saxton equation describes the geodesic flow on a sphere. J. Geom. Phys. 57, 2049-2064 (2007)

[24] Lenells, J.: Weak geodesic flow and global solutions of the Hunter-Saxton equation. Disc. Cont. Dyn. Syst. 18, 643-656 (2007)

[25] Lenells, J.: The Hunter-Saxton equation: A geometric approach. SIAM J. Math. Anal. 40, no. 1, 266-277 (2008)

[26] Lenells, J., Misiołek, G., Preston, S.C.: Curvatures of right-invariant Sobolev metrics on diffeomorphism groups and their Euler-Arnold equations, Preprint 2009

[27] Lenells, J., Misiołek, G., Tiğlay, F.: Integrable evolution equations on spaces of tensor densities and their peakon solutions. Commun. Math. Phys. 299 (2010) 129-161

[28] Liu, J., Yin, Z.: Global weak solutions for a periodic two-component $\mu$-HunterSaxton system. arXiv:1012.5452v3 [math.AP]

[29] McKean, H.P.: Curvature of an $\infty$-dimensional manifold related to Hill's equation. J. Differential Geom. 17(4) 523-529 (1982)

[30] Misiołek, G.: A shallow water equation as a geodesic flow on the Bott-Virasoro group. J. Geom. Phys. 24, 203-208 (1998)

[31] Misiołek, G.: Classical solutions of the periodic Camassa-Holm equation. GAFA 12 (2002), 1080-1104

[32] Okamoto, H.: Well-posedness of the generalized Proudman-Johnson equation without viscosity. J. Math. Fluid Mech. 11, 46-59 (2009)

[33] Pavlov, M.V.: The Gurevich-Zybin system. J. Phys. A: Math. Gen. 38, 38233840 (2005)

[34] Proudman, I., Johnson, K.: Boundary-layer growth near a rear stagnation point. J. Fluid Mech. 12, 161-168 (1962)

[35] Wunsch, M.: The generalized Hunter-Saxton system. SIAM J. Math. Anal. 42, no. 3, 1286-1304 (2010)

[36] Wunsch, M.: Weak geodesic flow on a semi-direct product and global solutions to the periodic Hunter-Saxton system. arXiv:1101.5483v1 [math.AP] 
[37] Yin, Z.: On the structure of solutions to the periodic Hunter-Saxton equation. SIAM J. Math. Anal. 36, no. 1, 272-283 (2004)

[38] Zou, D.: A two-component $\mu$-Hunter-Saxton equation. Inverse Problems 26 (2010) 085003 (9 pp.)

Institute for Applied Mathematics, University of Hannover, D-30167 Hannover, GerMANY

E-mail address: kohlmann@ifam.uni-hannover.de 